\title{
Identifying Intestinal Metaplasia at the Squamocolumnar Junction by Using Optical Coherence Tomography
}

\section{Citation}

Evans, John A., Brett E. Bouma, Jason Bressner, Milen Shishkov, Gregory Y. Lauwers, Mari MinoKenudson, Norman S. Nishioka, and Guillermo J. Tearney. 2007. Identifying intestinal metaplasia at the squamocolumnar junction by using optical coherence tomography. Gastrointestinal Endoscopy 65(1): 50-56.

\section{Published Version}

doi:10.1016/j.gie.2006.04.027

\section{Permanent link}

http://nrs.harvard.edu/urn-3:HUL.InstRepos:12601540

\section{Terms of Use}

This article was downloaded from Harvard University's DASH repository, and is made available under the terms and conditions applicable to Other Posted Material, as set forth at http:// nrs.harvard.edu/urn-3:HUL.InstRepos:dash.current.terms-of-use\#LAA

\section{Share Your Story}

The Harvard community has made this article openly available.

Please share how this access benefits you. Submit a story.

\section{Accessibility}




\title{
Identifying intestinal metaplasia at the squamocolumnar junction by using optical coherence tomography
}

\author{
John A. Evans, MD, Brett E. Bouma, PhD, Jason Bressner, BS, Milen Shishkov, PhD, Gregory \\ Y. Lauwers, MD, Mari Mino-Kenudson, MD, Norman S. Nishioka, MD, and Guillermo J. \\ Tearney, MD, PhD \\ Boston, Massachusetts, USA
}

\begin{abstract}
Background-Optical coherence tomography (OCT) is an optical imaging method that produces high-resolution cross-sectional images of the esophagus. The accuracy of OCT for differentiating tissue types at the squamocolumnar junction (SCJ) has not been established.
\end{abstract}

Objective-The purpose of this study was to identify and validate OCT image criteria for distinguishing metaplastic from nonmetaplastic tissue at the SCJ.

Design-A total of 196 biopsy-correlated OCT images of the SCJ were acquired from 113 patients undergoing upper endoscopy. A pathologist blinded to the OCT results reviewed each pathology specimen and determined the presence of the following histopathology: gastric cardia, squamous mucosa, pancreatic metaplasia, and intestinal metaplasia. An algorithm for diagnosing specialized intestinal metaplasia (SIM) was created by reviewing a training set of 40 biopsy-correlated OCT images. Two blinded investigators prospectively tested the algorithm on a validation set of 123 images.

Results-OCT images of squamous mucosa were characterized by a layered appearance without epithelial glands; gastric cardia, by vertical pit and gland structure, a well-defined epithelial surface reflectivity, and relatively poor image penetration; and SIM by an irregular architecture and good image penetration. The OCT criteria were $85 \%$ sensitive and 95\% specific for SIM when applied retrospectively to the training set. When applied to the validation set, the algorithm was $81 \%$ sensitive for both OCT readers and $66 \%$ and $57 \%$ specific for diagnosing SIM. The interobserver agreement was $\operatorname{good}(\kappa=0.53)$.

Conclusions-OCT imaging can identify SIM at the SCJ with an accuracy similar to that of endoscopy.

GERD is a well-known risk factor for the development of specialized intestinal metaplasia (SIM) of the esophagus, commonly known as Barrett's esophagus (BE). ${ }^{1}$ The prevalence of SIM has been estimated to be as high as $10 \%$ to $15 \%$ in patients with chronic GERD. ${ }^{2}$ For a patient with recurrent and severe symptoms of GERD, the adjusted odds ratio for developing adenocarcinoma over a 20-year period is 7.7 and 43.5 , respectively. ${ }^{3}$ Moreover, the incidence of esophageal adenocarcinoma and proximal stomach (gastric cardia) cancers have rapidly increased during the last 30 years. ${ }^{4-6}$ Because of the recognition of GERD as a risk factor for

Copyright $@ 2007$ by the American Society for Gastrointestinal Endoscopy

Reprint requests: Guillermo J. Tearney, MD, PhD, Harvard Medical School, Massachusetts General Hospital, Department of Pathology, Wellman Center for Photomedicine, 40 Blossom St, BAR703, Boston, MA 02114.

Current affiliations Gastrointestinal Unit (J.A.E., N.S.N.), Wellman Center for Photomedicine (B.E.B., J.B., M.S., G.J.T.), and

Department of Pathology (G.Y.L., M.M-K., G.J.T.), Massachusetts General Hospital, Boston, Massachusetts, USA

DISCLOSURE The authors have no conflicts of interest to disclose. 
developing esophageal cancer, upper endoscopic screening is recommended for white, male patients older than 50 years who have had chronic symptoms of GERD for more than 5 years. ${ }^{7}$ Endoscopic evaluation of the esophagus, with subsequent biopsy of regions suspicious for being metaplastic, is the only accepted method for diagnosing BE. The accuracy of this method is not ideal. Endoscopists correctly identify SIM in only $40 \%$ of cases, and the fractional area of tissue sampled by biopsy is small. ${ }^{8,9}$ A medical decision analysis performed to assess the cost-effectiveness of endoscopy for screening for SIM and high-grade dysplasia in patients with GERD concluded that the expense of endoscopy was one of the most important factors when determining whether or not screening was cost effective. ${ }^{10}$ This analysis found that screening for SIM would be justified if the cost of the screening method was significantly lower than the present cost of endoscopy. ${ }^{10}$ Alternative screening methods that provide greater esophageal-area coverage than conventional biopsy should improve the accuracy of detecting SIM. Furthermore, new methods carry the potential to reduce cost, thus partially alleviating the financial burden of comprehensive screening on the health care system.

Optical coherence tomography (OCT) is an optical imaging modality that uses near-infrared light to produce high-resolution (10- $\mu \mathrm{m}$ axial resolution) cross-sectional images of GI mucosa. Images are constructed based on light reflectivity in relation to the properties of the substrate being visualized. OCT can readily identify structures on a microscopic scale, including mucosal layers, "pit and gland" morphology, and glandular structure. ${ }^{11,12}$ A previous study demonstrated that OCT can distinguish SIM from squamous, fundic, and antral mucosa but can falsely identify gastric cardia as SIM. ${ }^{13}$ This prior study used biopsy-correlated images obtained throughout the upper-GI tract and as a result did not address the accuracy of OCT specifically at the squamocolumnar junction (SCJ), which is the region where SIM is thought to originate.

Four mucosal types typically populate the SCJ: gastric cardia or oxyntocardia, serous pancreatic metaplasia, squamous epithelia, and SIM. ${ }^{14,15}$ For OCT to be a reliable, sensitive, and cost-effective screening instrument, characterization of epithelial architecture at the SCJ should be accurate enough to distinguish premalignant (SIM) from benign tissue. The aim of this study was to identify and validate OCT image criteria of SIM, permitting its distinction from nonmetaplastic tissue at the gastroesophageal junction.

\section{PATIENTS AND METHODS}

\section{Design}

The study was a blinded, prospective trial. Its primary objective was to identify and validate OCT image features for differentiating intestinal metaplasia at the SCJ. Patients who were undergoing routine outpatient upper endoscopy were asked to participate in the study. OCT images of the SCJ were obtained during endoscopy. Two pathologists reviewed each biopsy specimen and noted the presence of the following tissue types: gastric or oxyntic cardia, squamous mucosa, or pancreatic metaplasia. The existence of intestinal metaplasia was characterized histologically by the presence of goblet cells. OCT image features of intestinal metaplasia were determined by creating and reviewing an OCT "training set," which contained biopsy-correlated images of known tissue types. These features were then prospectively applied to a "validation set" of unknown tissue types. The sensitivity, the specificity, and the reproducibility of the image criteria for diagnosis of intestinal metaplasia were determined.

\section{OCT system}

The OCT device was described in previous studies. ${ }^{13,16}$ The light source center wavelength was $1300 \mathrm{~nm}$, and the optical power incident on the tissue was $5.0 \mathrm{~mW}$. The spectral bandwidth of the source was $70 \mathrm{~nm}$, which provided an axial resolution of $10 \mu \mathrm{m}$. The catheter diameter 
was $2.5 \mathrm{~mm}$. Images were acquired in a linear longitudinal plane, with dimensions of $5.5 \mathrm{~mm}$ (1000 pixels) in length and $2.5 \mathrm{~mm}$ (500 pixels) in depth. During image acquisition, frames were recorded at a rate of 2 per second and were numbered sequentially for reference. A visible aiming laser coincident with the imaging beam allowed the endoscopist to localize the site of mucosa undergoing image acquisition, which facilitated biopsy correlation of the imaged site.

\section{Endoscopy and subject recruitment}

The protocol was reviewed and approved by the institutional review board at Massachusetts General Hospital. Recruited subjects included patients who were undergoing routine upper endoscopy and patients with known short-segment $(<1 \mathrm{~cm})$ intestinal metaplasia without known dysplasia at the gastroesophageal junction. Data were collected between November 2003 and September 2004. Subjects underwent routine upper endoscopy with conscious sedation and oropharyngeal anesthesia. A standard gastroscope (model EG 3470K; Pentax Medical, Tokyo, Japan), with a 3.8-mm instrument channel, was used.

\section{OCT imaging}

Written informed consent was obtained before the procedure. After adequate sedation and oropharyngeal anesthesia were achieved, an upper endoscopy was performed. The endoscopist identified the SCJ at the gastroesophageal junction or Barrett's segment. An OCT catheter probe was introduced through the instrument channel of the endoscope and advanced to the SCJ. Immediately distal to the SCJ, OCT images were acquired and recorded at the mucosal site marked by the visible aiming beam, and 1 jumbo biopsy specimen (with a 3.3-mm diameter biopsy forceps; Microvasive Endoscopy, Boston Scientific Corp. Natick, Mass) was obtained. OCT frames that corresponded to the imaged site were documented. Two biopsy-correlated images per patient were obtained. Acquisition of a biopsy-correlated image required approximately 30 seconds.

\section{Histopathology}

The biopsy specimens were placed in $10 \%$ formalin, embedded in paraffin, processed routinely, and stained with H\&E.

\section{Description of pathology review}

A pathologist reviewed each biopsy specimen and determined the presence of the following epithelial types: gastric cardia, squamous mucosa, serous pancreatic metaplasia, and SIM. A single diagnosis was rendered for biopsy specimens that contained multiple histologic types. For the purposes of this study, cardia mucosa and oxyntocardiac mucosa were grouped together as gastric cardia.

\section{OCT image analysis}

A "training set" that consisted of 20 randomly selected biopsy-correlated images of SIM and 20 randomly selected biopsy-correlated images of other tissue types were created. Two investigators (J.A.E., G.J.T.) familiar with OCT reviewed the training set and determined diagnostic image criteria for SIM. These criteria were then prospectively applied to a "validation set" that comprised the remainder of the data set. All OCT images in the validation set were reviewed to ensure image clarity, stripped of identifying information, and randomly allocated by investigators (J.B., B.B.) not involved with the determination of the diagnostic criteria. Images were removed from the data set if they were determined to be of poor quality, demonstrated linear artifacts caused by friction encountered by the OCT beam-scanning device, or failed to display adequate tissue within the image. 


\section{RESULTS}

\section{Training set}

The histopathologic diagnoses of the training set are shown in Table 1. Squamous epithelium was distinguished by a layered epithelium without glands (Fig. 1A). Gastric cardia was characterized by the presence of "pit and gland" morphology, regular surface architecture, the presence of a highly reflecting epithelial surface, or poor image penetration (Fig. 1B). For cases with a layered architecture, SIM was distinguished by the presence of epithelial glands (Fig. 2). In cases without layered architecture and "pit and gland" morphology, irregular surface architecture, lack of a highly reflecting epithelial surface, or good light penetration further differentiated SIM from the columnar epithelium of gastric cardia and ectopic pancreas (Fig. 3). A diagnostic algorithm for identifying SIM at the SCJ was formulated with these image criteria (Fig. 4).

When the diagnostic algorithm was retrospectively applied to the training set, it was $85 \%$ sensitive (95\% confidence interval [CI] 75\%-95\%) and 95\% specific (95\% CI 88\%-100\%) for differentiating SIM from nonmetaplastic tissue at the SCJ.

\section{Validation set}

Of the 156 biopsy-correlated images that comprised the validation set, 33 were excluded because of poor image quality, leaving a total of 123 biopsy-correlated images for prospective analysis. The histopathology included in the validation set and excluded because of inadequate image quality are detailed in Table 2 . When 2 blinded OCT readers applied the diagnostic flow chart (Fig. 4) to the validation set, the algorithm was found to be $81 \%$ (95\% CI 58\%-95\%) and $81 \%$ (95\% CI 58\%-95\%) sensitive, and 66\% (95\% CI 56\%-75\%) and 57\% (95\% CI 47\%-67\%) specific for a diagnosis of SIM at the SCJ. The agreement between the 2 readers was good $(\kappa=0.53)$. The truth tables for the 2 OCT readers are depicted in Table 3. The histologic breakdown of the 79 false-positive and the 125 true-negative results is presented in Table 4 . The histopathology of images when discrepant diagnoses were rendered by the OCT readers is described in Table 5.

\section{DISCUSSION}

This study identified specific OCT image characteristics that can distinguish intestinal metaplasia from benign, normal mucosa at the SCJ in a cohort of patients undergoing routine upper endoscopy. Although there is moderate variability in this distinction, a diagnostic sensitivity of $81 \%$ for SIM compares favorably with that of published endoscopic sensitivities $(82 \%) .{ }^{17}$ Our results strengthen the growing body of literature that describes the ability of OCT to distinguish tissue types on a histologic scale. ${ }^{18,19}$

The diagnostic flow chart accurately identified SIM when retrospectively applied to the training set, but the accuracy declined when prospectively applied to the validation set. Potential explanations of this difference in accuracy are inadequate tissue representation with the training set, registration error, and architectural distortion. The histologic diagnosis of SIM requires the identification of goblet cells on routine H\&E staining of esophageal biopsy specimens. Because current OCT systems cannot resolve individual goblet cells, the image characteristics of intestinal metaplasia based on tissue architecture needed to be determined. Gastric cardia and SIM comprised $17.5 \%$ and $50 \%$ of the training set, respectively. The analyzed validation set, however, contained $29 \%$ and $17 \%$ of gastric cardia and of SIM, respectively. It is possible that the training set did not provide an adequate representation of the variability of gastric cardia images, thereby reducing the overall OCT image accuracy. The accuracy of registration of biopsy sampling and image acquisition during the endoscopic procedure is difficult to 
estimate. Errors on the order of a few millimeters were likely and may have contributed to misdiagnosis by OCT. Histologic architectural changes, although sensitive for intestinal metaplasia, are not perfectly accurate; are seen in nonmetaplastic columnar mucosa; and when solely relied upon for a histologic diagnosis, could lead to significant errors in accuracy. Support for this hypothesis is shown in Table 4, which shows that gastric cardia $(\mathrm{n}=31)$ and columnar epithelia $(n=24)$ tissue types accounted for the majority $(70 \%)$ of false positives. Precision of SIM diagnosis between readers, however, was excellent. A discrepant diagnosis of SIM was rendered only twice, whereas columnar epithelia (cardia and columnar) for histologic diagnosis accounted for 7 and 12 discrepant diagnoses (Table 5).

The majority of images removed from the validation set were those that corresponded to a histologic diagnosis of the SCJ and the cardia (Table 2). Reasons for poor image acquisition of these areas are not completely understood but may be due to physical constraints of the OCT catheter within the working channel as torque is applied to the endoscope. Motion artifact from a moving esophagus is also a potential source of image distortion. Further study into OCT imaging of the SCJ is ongoing and may provide additional insights. Efforts to improve the OCT devices to minimize the percentage of poor quality images are also necessary.

There was good agreement between the observers' diagnoses of intestinal metaplasia $(\kappa=0.53)$, though there are no published data quantifying interobserver agreement of OCT image interpretation in the esophagus. For this study, we attempted to generate relatively simple diagnostic criteria to maintain reproducibility. It is possible that greater agreement between OCT and histopathology could arise from a more intricate scoring system. Future studies with more complex diagnostic criteria and larger data sets may be warranted to improve the accuracy of this technique.

A previous study examined OCT image features of SIM, squamous mucosa, and gastric mucosa. ${ }^{13}$ In contrast to this previous study, where tissue samples were taken randomly from the esophagus and the stomach, biopsy-correlated images in our study were performed only at the SCJ, thereby including a much higher proportion of image samples of gastric cardia and columnar epithelium. The proportional increase in columnar epithelium in this data set likely accounts for the differences in the determined sensitivities for SIM. Our study supports and confirms the ability of OCT to distinguish SIM by using a simple subjective visual assessment. This is a significant step in the application of OCT as a screening technique in the treatment and care of patients with GERD.

Before broad acceptance of OCT as a valid screening technique for detecting SIM in patients with GERD, further research in 3 areas will be needed. First, a system must be developed to deliver the OCT catheter to the esophageal lining in a simple manner that is comfortable to the patient and applicable to a large population. Second, dysplasia in BE will need to be easily and accurately identifiable. Third, computer-software-aided image processing and classification algorithms to allow easier interpretation of data by the practicing gastroenterologist will need to be generated. Finally, these applications and results must be reproducible on a large scale. Current OCT technology is rapidly advancing. High-resolution systems that produce better than 5-mm resolution are on the horizon. ${ }^{20,21}$ If these efforts are successful and if the criteria are sufficiently accurate, OCT may be used to comprehensively screen the SCJ without endoscopy, thus avoiding the costs of endoscopy, biopsy preparation, and pathology review. These technologic improvements may propel OCT to the forefront of optical biopsy technologies as a first-line screening technique in the management of GERD.

\section{Capsule Summary}




\section{What is already known on this topic}

-OCT produces high-resolution, cross-sectional images of the esophagus, but its accuracy for differentiating tissue types at the SCJ has not been established.

\section{What this study adds to our knowledge}

-In a blinded prospective study that used an algorithm for diagnosing SIM and a validation set of 123 biopsy-correlated OCT images, a diagnostic sensitivity of $81 \%$ for SIM indicated that OCT imaging can identify SIM at the SCJ with accuracy similar to that of endoscopy.

\section{New Online - Tell Us what You Think}

New Online Poling at www.giejoutnal.org. We want your opinion on imports journal topics. Beginning in April 2006, we are posting online survey questions that ask contents, value and other journal-related questions. Check the GIE homepage periodically for new questions and polling results to see what your colleagues are saying about Gastrointestinal Endoscopy.

GIE Online Polling at www.giejournal.org

\section{ACKNOWLEDGMENTS}

The authors would like to thank William Puricelli, RN, clinical research coordinator, for his support.

This study was funded, in part, by the Center for Integration of Innovative Technologies and Medicine (CIMIT, development of the imaging platform) and the National Institutes of Health (NIH-R01 CA 103769).

\section{REFERENCES}

1. Loffeld RJ, van der Putten AB. Rising incidence of reflux oesophagitis in patients undergoing upper gastrointestinal endoscopy. Digestion 2003;68:141-4. [PubMed: 14646335]

2. Winters C, Spurling T, Chobanian S, et al. Barrett's esophagus. A prevalent, occult complication of gastroesophageal reflux disease. Gastroenterology 1987;92:118-24. [PubMed: 3781178]

3. Lagergren J, Bergstrom R, Lindgren A, et al. Symptomatic gastroesophageal reflux as a risk factor for esophageal adenocarcinoma. N Engl J Med 1999;340:825-31. [PubMed: 10080844]

4. Blot WJ, Devesa SS, Kneller RW, et al. Rising incidence of adenocarcinoma of the esophagus and gastric cardia. JAMA 1991;265:1287-9. [PubMed: 1995976]

5. Bytzer P, Christensen PB, Damkier P, et al. Adenocarcinoma of the esophagus and Barrett's esophagus: a population-based study. Am J Gastroenterol 1999;94:86-91. [PubMed: 9934736]

6. Devesa SS, Blot WJ, Fraumeni JF Jr. Changing patterns in the incidence of esophageal and gastric carcinoma in the United States. Cancer 1998;83:2049-53. [PubMed: 9827707]

7. Spechler SJ. Screening and surveillance for complications related to gastroesophageal reflux disease. Am J Med 2001;111(Suppl 8A):130S-6S. [PubMed: 11749938]

8. Falk GW, Rice TW, Goldblum JR, et al. Jumbo biopsy forceps protocol still misses unsuspected cancer in Barrett's esophagus with high-grade dysplasia. Gastrointest Endosc 1999;49:170-6. [PubMed: 9925694]

9. Cameron AJ, Carpenter HA. Barrett's esophagus, high-grade dysplasia, and early adenocarcinoma: a pathological study. Am J Gastroenterol 1997;92:586-91. [PubMed: 9128304]

10. Soni A, Sampliner RE, Sonnenberg A. Screening for high-grade dysplasia in gastroesophageal reflux disease: is it cost effective? Am J Gastroenterol 2000;95:2086-93. [PubMed: 10950062]

11. Westphal V, Rollins AM, Willis J, et al. Correlation of endoscopic optical coherence tomography with histology in the lower-GI tract. Gastrointest Endosc 2005;61:537-46. [PubMed: 15812406]

12. Brand S, Poneros JM, Bouma BE, et al. Optical coherence tomography in the gastrointestinal tract. Endoscopy 2000;32:796-803. [PubMed: 11068841] 
13. Poneros JM, Brand S, Bouma BE, et al. Diagnosis of specialized intestinal metaplasia by optical coherence tomography. Gastroenterology 2001;120:7-12. [PubMed: 11208708]

14. Chandrasoma PT, Der R, Dalton P, et al. Histology of the gastroesophageal junction: an autopsy study. Am J Surg Pathol 2000;24:402-9. [PubMed: 10716154]

15. Sarbia M, Donner A, Gabbert HE. Histopathology of the gastroesophageal junction: a study on 36 operation specimens. Am J Surg Pathol 2002;26:1207-12. [PubMed: 12218577]

16. Poneros JM, Tearney GJ, Shishkov M, et al. Optical coherence tomography of the biliary tree during ERCP. Gastrointest Endosc 2002;55:84-8. [PubMed: 11756925]

17. Eloubeidi MA, Provenzale D. Does this patient have Barrett's esophagus? The utility of predicting Barrett's esophagus at the index endoscopy. Am J Gastroenterol 1999;94:937-43. [PubMed: 10201460]

18. Shen B, Zuccaro G Jr, Gramlich TL, et al. In vivo colonoscopic optical coherence tomography for transmural inflammation in inflammatory bowel disease. Clin Gastroenterol Hepatol 2004;2:10807. [PubMed: 15625653]

19. Shen B, Zuccaro G Jr, Gramlich TL, et al. Ex vivo histology-correlated optical coherence tomography in the detection of transmural inflammation in Crohn's disease. Clin Gastroenterol Hepatol 2004;2:754-60. [PubMed: 15354275]

20. Herz P, Chen Y, Aguirre AD, et al. Micromotor endoscope catheter for in vivo, ultrahigh-resolution optical coherence tomography. Opt Lett 2004;29:2261-3. [PubMed: 15524374]

21. Oh NY, Yun SH, Tearney GJ, et al. Wide tuning range wavelength-swept laser with two semiconductor optical amplifiers. Phot Tech Lett 2005;17:6780-3. 


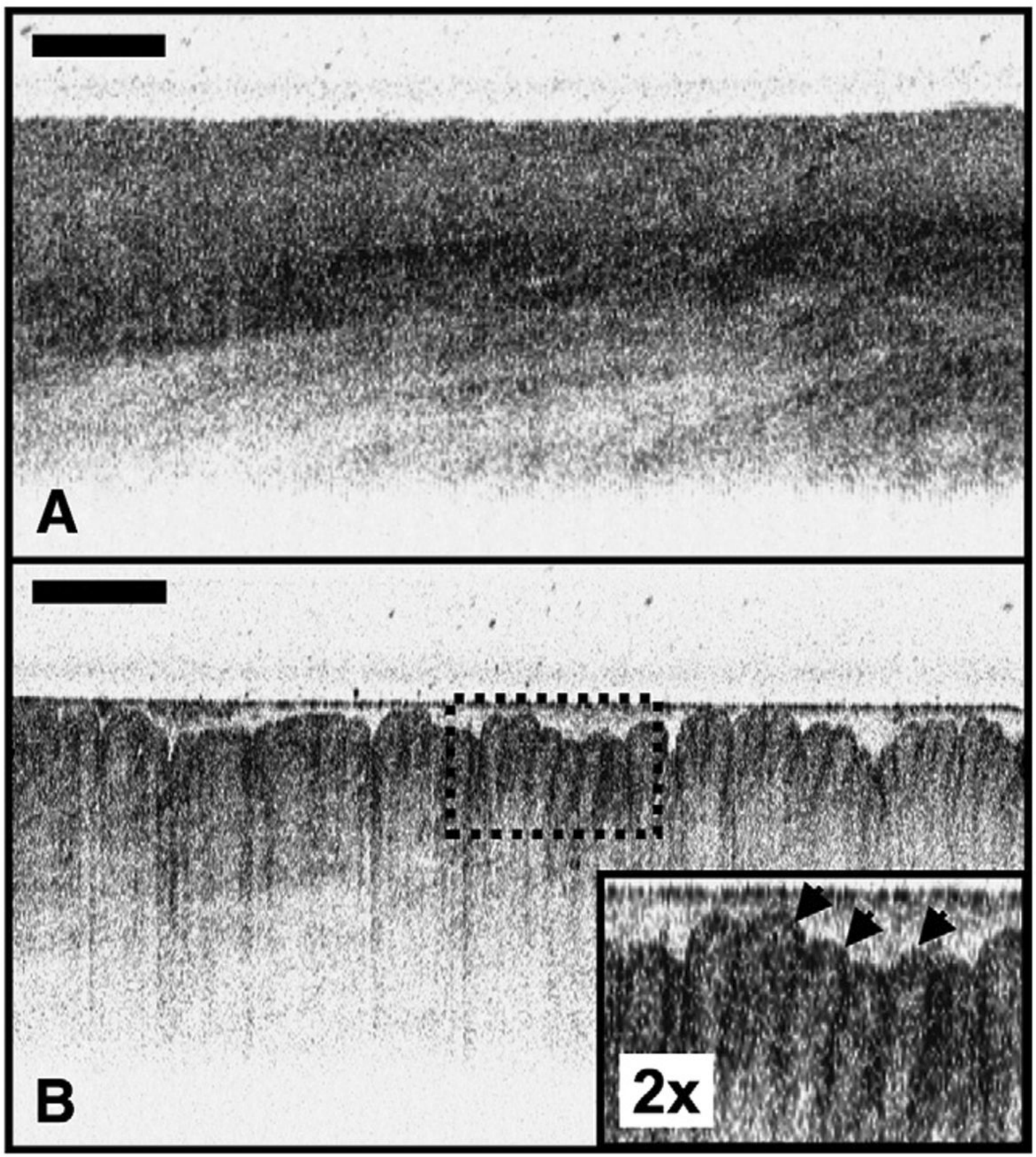

Figure 1.

Images of nonmetaplasic epithelium. A, OCT image of squamous epithelium demonstrates a horizontally layered architecture. B, OCT image of gastric cardia shows regular, vertical "pit and gland" architecture, a highly scattering epithelial surface, and relatively poor image penetration. Scale bars, $500 \mu \mathrm{m}$. 

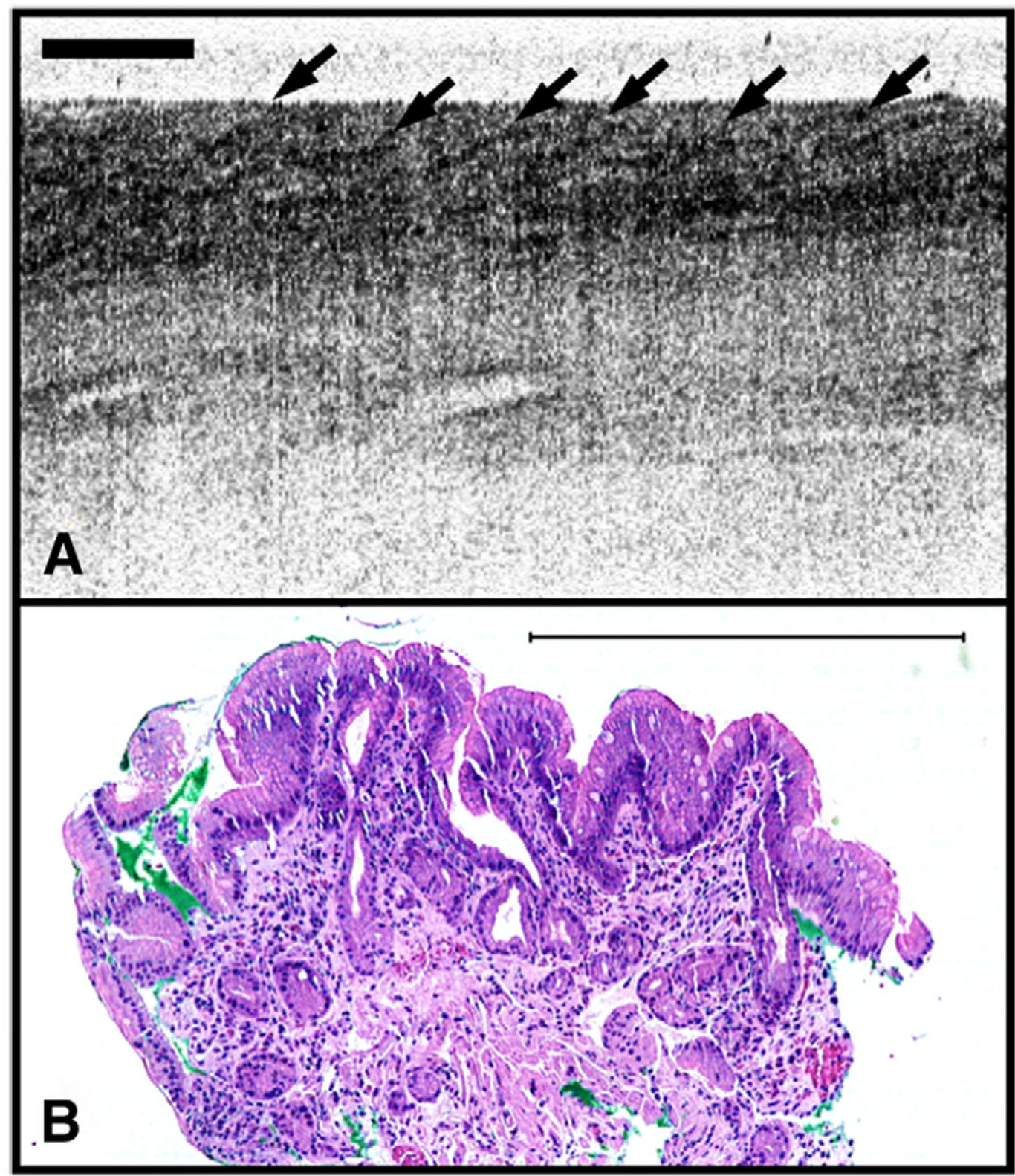

Figure 2.

Images of SIM with a layered architecture. A, Horizontal layered architecture can be visualized in this OCT image of SIM. Glands present in the superficial layer (arrows) differentiate this tissue from squamous epithelium. B, Corresponding histology (H\&E, orig. mag. $\times 100)$. Scale bars, $500 \mu \mathrm{m}$. 


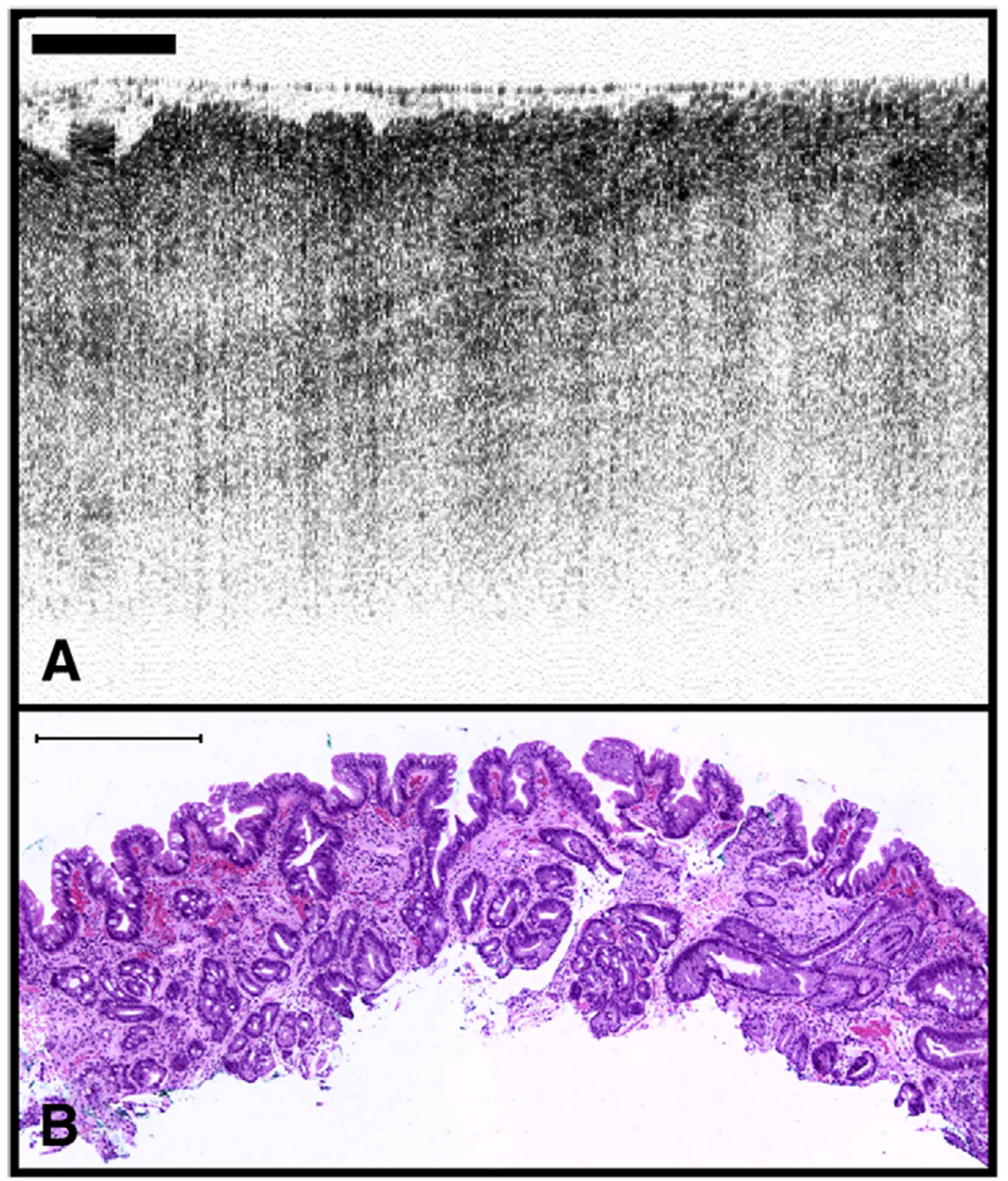

Figure 3.

Images of SIM without a layered architecture. A, Absence of a layered or regular "pit and gland" architecture, low superficial epithelial reflectivity, and relatively good image penetration are characteristic of SIM at the SCJ. B, Corresponding histology (H\&E, orig. mag. $\times 40)$. Scale bars, $500 \mu \mathrm{m}$. 


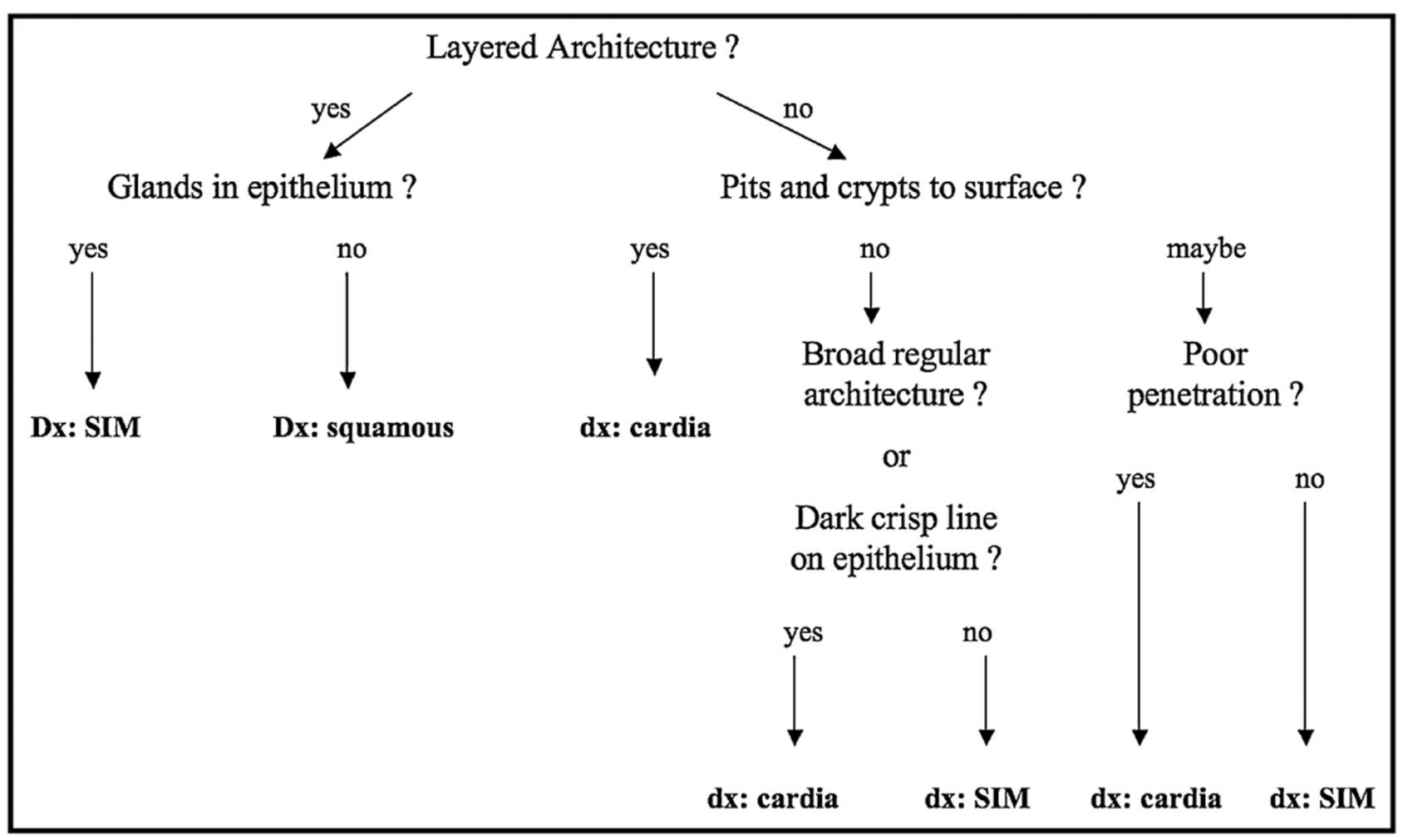

Figure 4.

Diagnostic algorithm flowchart for differentiating SIM at the SCJ. 
Histology breakdown of training set

\section{TABLE 1}

\begin{tabular}{lcc}
\hline Histology type & No. in training set & Percentage of whole \\
\hline Intestinal metaplasia & 20 & 50 \\
Gastric cardia & 7 & 50 \\
Squamous mucosa & 0 & 0 \\
Squamocolumnar & 10 & 25 \\
Columnar & 2 & 25 \\
Pancreatic tissue & 1 & 2.5 \\
Total & 40 & 100 \\
\hline
\end{tabular}

* Columnar epithelium, not metaplastic, not cardia. 
TABLE 2

Histology included and excluded from validation set

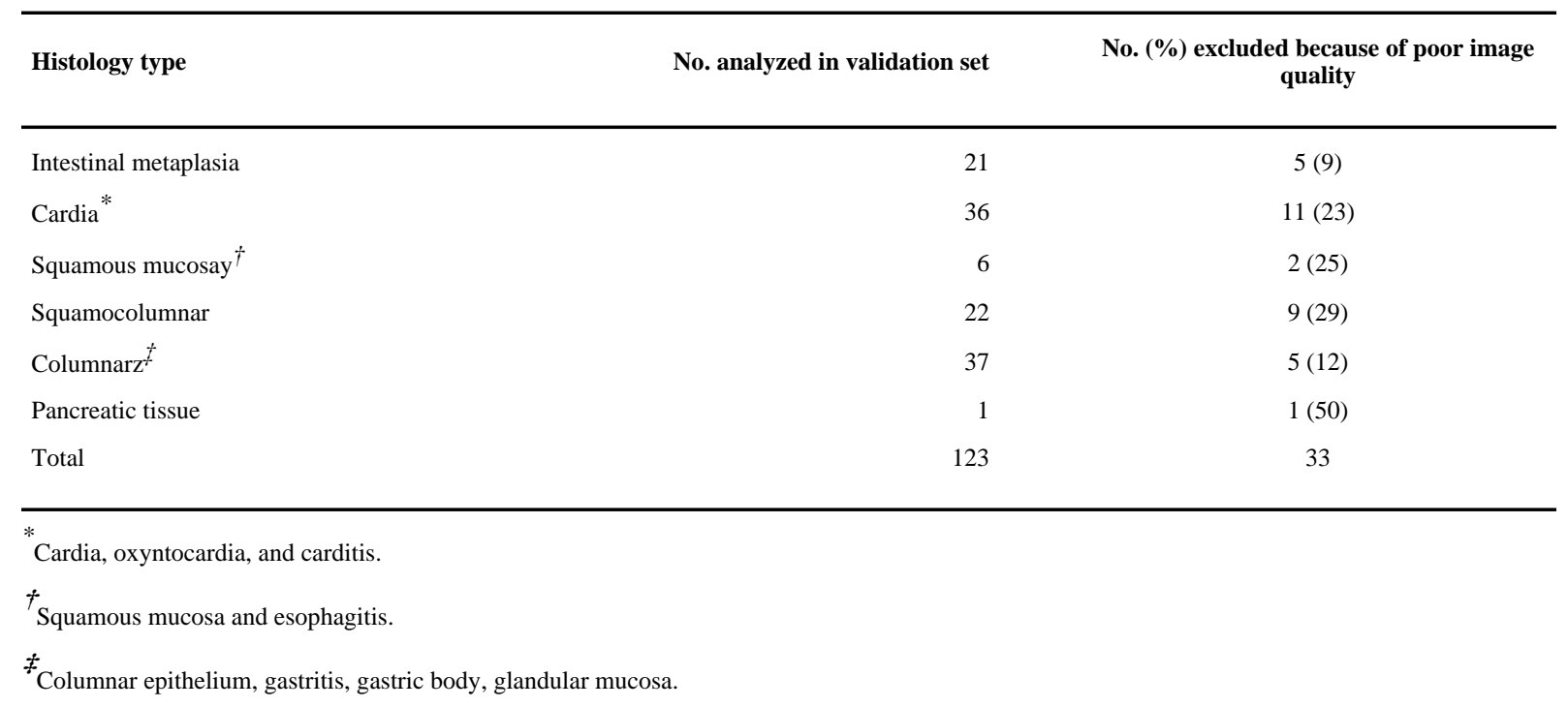


Truth tables

\section{TABLE 3}

\begin{tabular}{|c|c|c|c|c|}
\hline \multirow[b]{2}{*}{ Truth table } & & \multicolumn{2}{|c|}{ Pathologic diagnosis } & \multirow[b]{2}{*}{ Total } \\
\hline & & Nonmetaplastic & Intestinal metaplasia & \\
\hline \multicolumn{5}{|l|}{ GT truth table } \\
\hline \multirow[t]{3}{*}{$\begin{array}{l}\text { Diagnosis from OCT } \\
\text { image }\end{array}$} & Nonmetaplastic & 67 & 4 & 71 \\
\hline & Intestinal metaplasia & 35 & 17 & 52 \\
\hline & Total & 102 & 21 & 123 \\
\hline \multicolumn{5}{|l|}{ JE truth table } \\
\hline \multirow[t]{3}{*}{$\begin{array}{l}\text { Diagnosis from OCT } \\
\text { image }\end{array}$} & Nonmetaplastic & 58 & 4 & 62 \\
\hline & Intestinal metaplasia & 44 & 17 & 61 \\
\hline & Total & 102 & 21 & 123 \\
\hline
\end{tabular}

GT, Dr Tearney; JE, Dr Evans. 
TABLE 4

Histologic breakdown of false positives and true negatives

\begin{tabular}{lrr}
\hline & False positives & True negatives \\
\hline Cardia $^{*}$ & 31 & 41 \\
Squamous mucosa $^{\dagger}$ & 6 & 6 \\
Squamocolumnar & 16 & 28 \\
Columnar & 24 & 50 \\
Pancreatic tissue & 2 & 0 \\
Total & 79 & 125 \\
${ }^{*}$ Cardia, oxyntocardia, and carditis. & & \\
${ }^{\dagger}$ Squamous mucosa and esophagitis. & & \\
${ }^{*}$ Columnar epithelium, gastritis, gastric body, glandular mucosa. &
\end{tabular}


TABLE 5

Breakdown of discrepant diagnoses by OCT readers

Histology type

No. discrepant OCT diagnoses

Intestinal metaplasia

Cardia $^{*}$

Squamous mucosa ${ }^{\dagger}$

Squamocolumnar

Columnar ${ }^{\dagger}$

Pancreatic tissue

Total

* Cardia, oxyntocardia, and carditis.

${ }^{\dagger}$ Squamous mucosa and esophagitis.

${ }^{\ddagger}$ Columnar epithelium, gastritis, gastric body, glandular mucosa. 